\title{
Erratum to: Epimorphisms Between Linear Orders
}

\author{
Riccardo Camerlo ${ }^{1}$. Raphaël Carroy ${ }^{2}$. \\ Alberto Marcone $\mathbf{3}^{3}$
}

Published online: 27 October 2015

(C) Springer Science+Business Media Dordrecht 2015

\section{Erratum to: Order 32, 387-400 (2015) DOI 10.1007/s11083-014-9339-3}

After publication of our paper, we learned from Maurice Pouzet that one of our main results, namely Theorem 21, was already proved - with a different argument - in C. Landraitis: A combinatorial property of the homomorphism relation between countable order types, Journal of Symbolic Logic 44, 403-411 (1979).

The online version of the original article can be found at http://dx.doi.org/10.1007/s11083-014-9339-3.

Alberto Marcone

alberto.marcone@uniud.it

Riccardo Camerlo

riccardo.camerlo@polito.it

Raphaël Carroy

raphael.carroy@unito.it

1 Dipartimento di Scienze Matematiche “Joseph-Louis Lagrange”, Politecnico di Torino, Corso Duca degli Abruzzi 24, 10129 Torino, Italy

2 Dipartimento di Matematica “Giuseppe Peano”, Università di Torino, Via Carlo Alberto 10, 10121 Torino, Italy

3 Dipartimento di Matematica e Informatica, Università di Udine, Via delle Scienze 208, 33100 Udine, Italy 